\title{
Spectrophotometry of star forming regions in HII galaxies
}

\author{
Carolina Kehrig ${ }^{1}$, Eduardo Telles ${ }^{1}$ and François Cuisinier ${ }^{2}$ \\ ${ }^{1}$ Observatório Nacional, Rio de Janeiro, Brazil email: kehrig@on.br, etelles@on.br \\ ${ }^{2}$ Departamento de Astronomia, Universidade Federal do Rio de Janeiro, Brazil \\ email: francois@ov.ufrj.br
}

\begin{abstract}
We present the results of a spectrophotometric study of star forming regions in HII galaxies. In particular, we find that, besides their "blobbiness", HII galaxies are quite homogenous in their chemical properties, though we do see important fluctuations in some physical properties (e.g. extinction).
\end{abstract}

We present spectrophotometric observations of 111 HII galaxies, performed on the ESO $1.52 \mathrm{~m}$ telescope. This sample includes many galaxies from Terlevich et al. 1991 (hereafter T91) with additional targets from the Calan-Tololo (Maza et al. 1989) and Marseille (Surace \& Comte 1998) surveys, observed and analysed in a homogeneous way (Kehrig et al. 2004). Here we analyse their statistical properties and derive physical conditions, as well as chemical abundances. Details of this analysis will appear in a forthcoming paper.

In order to evaluate the quality of our data, we compare our emission line intensities with T91 for galaxies in common in both datasets. The two datasets agree well within 0.1 dex. For low intensity lines, T91 measurements are systematically higher than ours, which may indicate higher $\mathrm{S} / \mathrm{N}$ in our spectra (Rola \& Pelat 1994). This allowed us to measure low intensity lines in more objects, other emission lines not measured before, as well as a better estimate of the continuum and some absorption features (Westera et al. 2004).

Oxygen abundances $(\mathrm{O} / \mathrm{H})$ were derived by 4 different methods. The "direct" method (or the classic Te-method) and 3 strong line methods. The P-method (Pilyugin 1991) presented the better agreement with the "direct" method. In Figure 1 (left panel) we see the thick solid line histogram (P-method) extends to $\mathrm{O} / \mathrm{H}$ greater than the hatched histogram (Te-method) indicating that when we use the "direct" method we can be missing the high abundance end, due to an observational bias.

We have also spatially resolved distinct regions of star formation in individual galaxies and determined the spectroscopic properties for these regions separately. The variations of $\mathrm{O} / \mathrm{H}$ between the individual regions are small and within observational uncertainties. This result does not depend on the method used to derive the abundances (Figure 1 - right panel). Thus, our main result is that, despite their "blobbiness" and significant variations in some physical properties (ex: extinction), HII galaxies are chemically homogeneous and well mixed.

We have also constructed a series of evolutionary diagrams using emission lines ratios and $\mathrm{EW}(\mathrm{H} \beta)$, and interpreted them with photoionization models for single stellar populations (Stasinska et al. 2001) (Figure 2). We verified that HII galaxies do not correspond to the simplistic view of instantaneous bursts. The observed relations can be better understood if most HII galaxies contain older stellar populations. This result agrees with those obtained by Westera et al. (2004). In this work the stellar content of our sample of HII galaxies was analysed through spectral indices. We found that all galaxies contain 

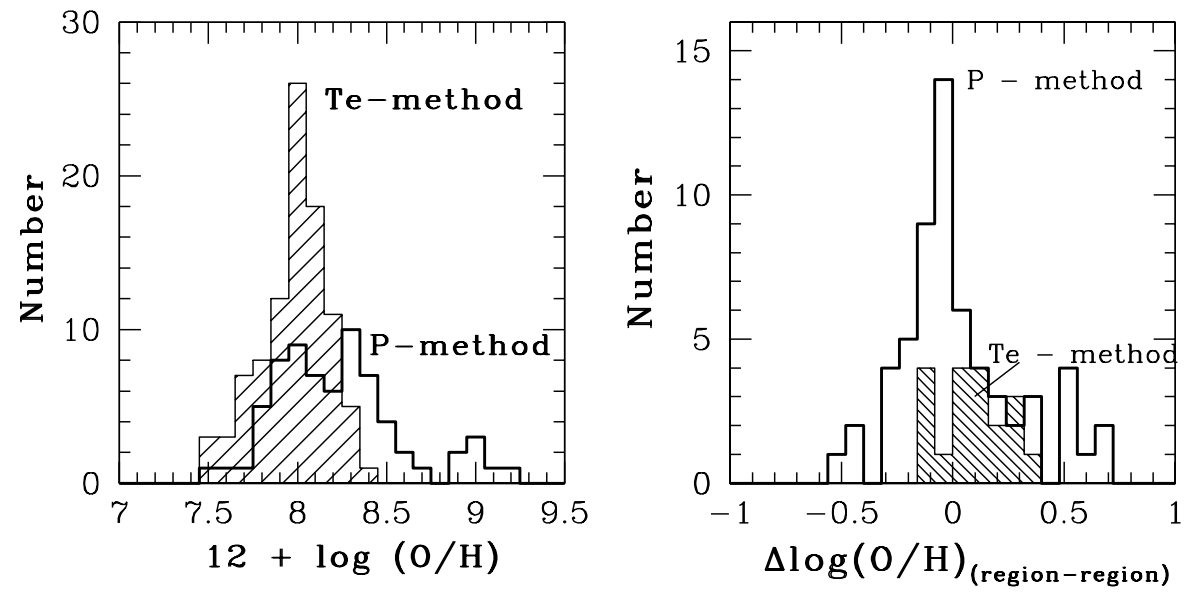

Figure 1. Left: $\mathrm{O} / \mathrm{H}$ distribution determined by two different methods. Right: Comparison between oxygen abundances of individual regions.

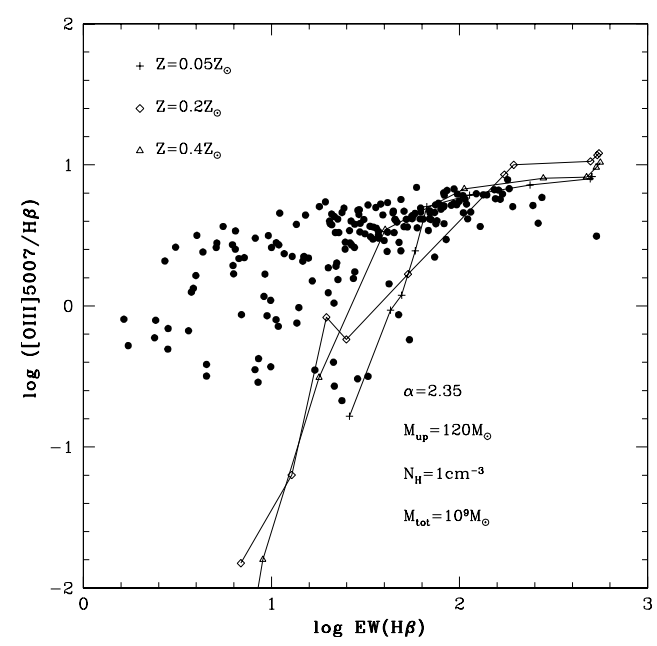

Figure 2. $[\mathrm{OIII}] / \mathrm{H} \beta$ line ratio vs. equivalent width of $\mathrm{H} \beta$. Lines represent Single Stellar Population models from Stasinska et al. 2001

underlying stellar population from previous star formation events, which were more important in turning the gas into stars than the present burst.

\section{References}

Kehrig, C., Telles, E., \& Cuisinier, F. 2004, AJ, submitted

Maza, J., Ruiz, M. T., Gonzàlez, L. E., \& Wichjniesky, M. 1989, ApJS, 69, 349

Pilyugin, L. S. 1991, A\&A, 362, 325

Rola, C., \& Pelat, D. 1970, A\&A, 282, 199

Stasinska, G., Schaerer, D., \& Leitherer, C. 2001, A\&A, 370, 1

Surace, C., \& Comte, G. 1998, A\&AS, 133, 171

Terlevich, R., et al. 1991, A\&AS, 91, 285

Westera, P., Cuisinier, F., Telles, E., \& Kehrig, C. 2004, A\&A, in press 